Document downloaded from:

http://hdl.handle.net/10251/99723

This paper must be cited as:

Hueso Pagoaga, JL.; Martínez Molada, E. (2014). Semilocal convergence of a family of iterative methods in Banach spaces. Numerical Algorithms. 67(2):365-384. doi:10.1007/s11075-013-9795-7

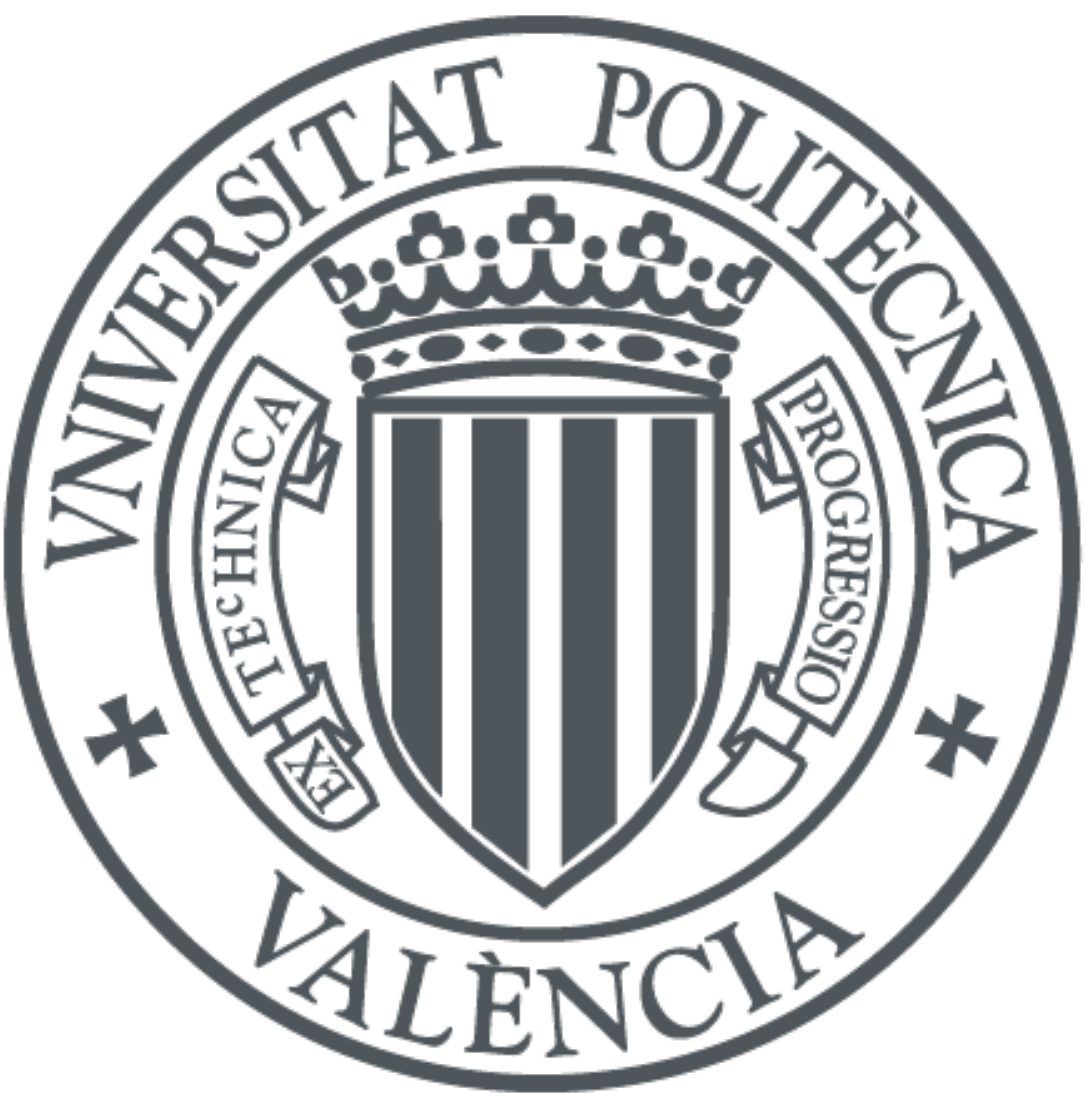

The final publication is available at

http://doi.org/10.1007/s11075-013-9795-7

Copyright Springer-Verlag

Additional Information 


\title{
Semilocal convergence of a family of iterative methods in Banach spaces
}

\author{
José L. Hueso • Eulalia Martínez
}

Received: date / Accepted: date

\begin{abstract}
In this work, we prove a third and fourth convergence order result for a family of iterative methods for solving nonlinear systems in Banach spaces. We analyze the semilocal convergence by using recurrence relations, given the existence and uniqueness theorem that established the R-order of the method and the priori error bounds. Finally, we apply the methods to two examples in order to illustrate the presented theory.
\end{abstract}

Keywords Nonlinear systems · Iterative method · Banach space · Recurrence relations $\cdot$ Semilocal convergence $\cdot$ R-order.

\section{Introduction}

Let us consider the problem of finding a zero of a nonlinear function $F: \Omega \subseteq X \longrightarrow Y$, on an open convex set $\Omega$ of a Banach space $X$ with values in a Banach space $Y$. Iterative methods are the most usual procedure to approximate a solution of the problem. The best known iterative method is the classical Newton's method, [1], whose semilocal convergence using recurrence relations was obtained by Kantorovich in [2].

The study of semilocal convergence for an iterative method in Banach spaces is very interesting because just imposing conditions on the starting point $x_{0}$, instead of

This work has been supported by Ministerio de Ciencia e Innovación MTM2011-28636-C02-02 and by Vicerrectorado de Investigación. Universitat Politècnica de València PAID-SP-20120498

José L. Hueso,

Instituto de Matemática Multidisciplinar,

E-mail: jlhueso@mat.upv.es

Eulalia Martínez

Instituto de Matemática Pura y Aplicada,

E-mail: eumarti@mat.upv.es

Universitat Politècnica de València,

Camino de Vera, s/n, 46022

Valencia, Spain 
on the solution, important results can be obtained, such as, existence and uniqueness of the solution, convergence order, a priori error bounds and convergence domains. These results can be applied to the solution of some practical problems described by differential equations, partial differential equations and integral equations.

In [3,4], Marquina et al., using recurrence relations, obtain semilocal convergence results for third order methods such as Chebyshev and Halley's methods.

This technique has been widely studied by Hernandez et al., [5,6] for establishing the semilocal convergence results simplifying the construction of the recurrence relations needed. Some variants in this procedure are proposed in [7]-[8]. Recently, semilocal convergence has been used to establish the R-order of higher order methods, [9]-[15]. Moreover, this technique is used for finding the domain of convergence under weaker assumptions [16]-[19].

In [20], the authors introduced a family of third and four order methods for nonlinear systems giving local convergence results. This family becomes very interesting in terms of efficiency, as it can be seen in the work, because it only uses the first Frechet-derivative. This is the reason why we want to deepen in its study.

Our aim in this paper is to establish the semilocal convergence for this family of iterative methods in Banach spaces and to derive the error estimations by constructing a system of recurrence relations.

In Section 2, we give some preliminary results and define the auxiliary functions. In Section 3, we construct the recurrence relations in order to establish the semilocal convergence, what is done in Section 4. Section 5 shows the application of the theoretical results to a pair of nonlinear systems of different size and Section 6 depicts results of global convergence in an example system.

\section{Preliminary results}

Let $X, Y$ be Banach spaces and $F: \Omega \subseteq X \rightarrow Y$ be a nonlinear twice Fréchet differentiable operator in an open convex domain $\Omega_{0} \subseteq \Omega$. From now on we will consider the iterative method given in [20], for solving the system $F(x)=0$ defined by

$$
\begin{aligned}
y_{n} & =x_{n}-a \Gamma_{n} F\left(x_{n}\right) \\
z_{n} & =x_{n}-\Gamma_{n}\left(F\left(y_{n}\right)+a F\left(x_{n}\right)\right) \\
x_{n+1} & =x_{n}-\Gamma_{n}\left(F\left(z_{n}\right)+F\left(y_{n}\right)+a F\left(x_{n}\right)\right),
\end{aligned}
$$

where $a \in \mathbf{R}$ and $\Gamma_{n}=F^{\prime}\left(x_{n}\right)^{-1}$.

Let $x_{0} \in \Omega_{0}$ such as $\Gamma_{0}=F^{\prime}\left(x_{0}\right)^{-1}$ exists and that

$$
\begin{aligned}
& \left\|\Gamma_{0}\right\| \leq \beta \\
& \left\|\Gamma_{0} F\left(x_{0}\right)\right\| \leq \eta \\
& \left\|F^{\prime \prime}(x)\right\| \leq M, \quad x \in \Omega_{0} \\
& \left\|F^{\prime \prime}(x)-F^{\prime \prime}(y)\right\| \leq K\|x-y\|, \quad x, y \in \Omega_{0} .
\end{aligned}
$$

Let us define $\beta_{0}=\left\|\Gamma_{0}\right\|, \eta_{0}=\left\|\Gamma_{0} F\left(x_{0}\right)\right\|, a_{0}=M \beta_{0} \eta_{0}$ and $b_{0}=K \beta_{0} \eta_{0}^{2}$. Observe that, using (1) one has

$$
\left\|y_{0}-x_{0}\right\|=\left\|-a \Gamma_{0} F\left(x_{0}\right)\right\|=|a| \eta_{0} .
$$


In the following lemma we give an expression and a bound of the remainder of the Taylor expansion of the operator $F$ which will be used in subsequent proofs.

Lemma 1 If $F$ has continuous derivatives up to order $k+1$ in a convex open set $\Omega$ and $\left\|F^{k+1}(x)\right\| \leq P$, for all $x \in \Omega$, then, if $x_{0} \in \Omega$,

$$
F(x)=\sum_{j=0}^{k} \frac{1}{k !} F^{(k)}\left(x_{0}\right)\left(x-x_{0}\right)^{k}+R_{x_{0}, k}\left(x-x_{0}\right),
$$

where the remainder can be expressed as

$$
R_{x_{0}, k}\left(x-x_{0}\right)=\frac{1}{k !} \int_{0}^{1}(1-t)^{k} F^{k+1}\left(x_{0}+t\left(x-x_{0}\right)\right)\left(x-x_{0}\right)^{k+1} d t
$$

and

$$
\left\|R_{x_{0}, k}\left(x-x_{0}\right)\right\| \leq \frac{P}{(k+1) !}\left\|x-x_{0}\right\|^{k+1} .
$$

The well known Banach's Lemma, [21], will be used to guarantee the existence and boundedness of the inverse of a matrix.

Lemma 2 (Banach's Lemma): Let $A \in \mathbb{L}(X, X)$ and $\|A\|<1$ then $I-A$ is an invertible matrix and $\left\|(I-A)^{-1}\right\|<\frac{1}{1-\|A\|}$.

We want to apply this result to $I-\Gamma_{0} F^{\prime}\left(x_{1}\right)$, in order to prove the existence of the inverse matrix of $\Gamma_{0} F^{\prime}\left(x_{1}\right)$ and then the inverse of $F^{\prime}\left(x_{1}\right)$. Then the following condition must be satisfied:

$$
\begin{aligned}
\left\|I-\Gamma_{0} F^{\prime}\left(x_{1}\right)\right\| & \leq\left\|\Gamma_{0}\right\|\left\|F^{\prime}\left(x_{0}\right)-F^{\prime}\left(x_{1}\right)\right\| \\
& \leq\left\|\Gamma_{0}\right\|\left\|\int_{x_{0}}^{x_{1}} F^{\prime \prime}(x) d x\right\| \leq \beta_{0} M\left\|x_{1}-x_{0}\right\|<1 .
\end{aligned}
$$

We need a bound for $\left\|x_{1}-x_{0}\right\|$. Using the triangular inequality, (3) and (2), we can write

$$
\begin{aligned}
\left\|x_{1}-x_{0}\right\| & \leq\left\|x_{1}-z_{0}\right\|+\left\|z_{0}-x_{0}\right\| \\
& =\left\|\Gamma_{0} F\left(z_{0}\right)\right\|+\left\|\Gamma_{0}\left(F\left(y_{0}\right)+a F\left(x_{0}\right)\right) .\right\|
\end{aligned}
$$

Consider the Taylor expansion

$$
\begin{aligned}
F\left(z_{0}\right) & =F\left(y_{0}\right)+F^{\prime}\left(y_{0}\right)\left(z_{0}-y_{0}\right)+R_{y_{0}, 1}\left(z_{0}-y_{0}\right) \\
& =F\left(y_{0}\right)+\left(F^{\prime}\left(y_{0}\right)-F^{\prime}\left(x_{0}\right)\right)\left(z_{0}-y_{0}\right) \\
& +F^{\prime}\left(x_{0}\right)\left(z_{0}-y_{0}\right)+R_{y_{0}, 1}\left(z_{0}-y_{0}\right)
\end{aligned}
$$

where $R_{y_{0}, 1}\left(z_{0}-y_{0}\right)$ is the remainder of the first degree expansion. Using (2), we substitute $F^{\prime}\left(x_{0}\right)\left(z_{0}-y_{0}\right)$ by $-F\left(y_{0}\right)$, obtaining

$$
\begin{aligned}
F\left(z_{0}\right) & =\left(F^{\prime}\left(y_{0}\right)-F^{\prime}\left(x_{0}\right)\right)\left(z_{0}-y_{0}\right)+R_{y_{0}, 1}\left(z_{0}-y_{0}\right) \\
& =\int_{x_{0}}^{y_{0}} F^{\prime \prime}(\tau)\left(z_{0}-y_{0}\right) \mathrm{d} \tau+R_{y_{0}, 1}\left(z_{0}-y_{0}\right) .
\end{aligned}
$$


Then, using (6) and Lemma 1 we get

$$
\left\|F\left(z_{0}\right)\right\| \leq M\left\|y_{0}-x_{0}\right\|\left\|z_{0}-y_{0}\right\|+\frac{1}{2} M\left\|z_{0}-y_{0}\right\|^{2} .
$$

Using (1), we have

$$
F^{\prime}\left(x_{0}\right)\left(y_{0}-x_{0}\right)=-a F\left(x_{0}\right),
$$

so that, the Taylor expansion

$$
F\left(y_{0}\right)=F\left(x_{0}\right)+F^{\prime}\left(x_{0}\right)\left(y_{0}-x_{0}\right)+R_{x_{0}, 1}\left(y_{0}-x_{0}\right) .
$$

can be written as

$$
F\left(y_{0}\right)=(1-a) F\left(x_{0}\right)+R_{x_{0}, 1}\left(y_{0}-x_{0}\right)
$$

and thus

$$
\Gamma_{0}\left(F\left(y_{0}\right)+a F\left(x_{0}\right)\right)=\Gamma_{0} F\left(x_{0}\right)+\Gamma_{0} R_{x_{0}, 1}\left(y_{0}-x_{0}\right) .
$$

Taking norms and using Lemma 1 and (8), we get

$$
\left\|z_{0}-x_{0}\right\|=\left\|\Gamma_{0}\left(F\left(y_{0}\right)+a F\left(x_{0}\right)\right)\right\| \leq \eta_{0}+\frac{M \beta_{0}}{2}\left\|y_{0}-x_{0}\right\|^{2}=\left(1+\frac{a_{0}}{2} a^{2}\right) \eta_{0} .
$$

From (1) and (12)

$$
\left\|z_{0}-y_{0}\right\|=\left\|-\Gamma_{0} F\left(y_{0}\right)\right\|=\left\|(1-a) \Gamma_{0} F\left(x_{0}\right)+\Gamma_{0} R_{x_{0}, 1}\left(y_{0}-x_{0}\right)\right\|,
$$

and using (4), Lemma 1 and (8) one has

$$
\left\|z_{0}-y_{0}\right\| \leq|1-a| \eta_{0}+\frac{1}{2} M \beta_{0} a^{2} \eta_{0}^{2}=\left(|1-a|+\frac{a_{0} a^{2}}{2}\right) \eta_{0}=q_{a}\left(a_{0}\right) \eta_{0},
$$

where

$$
q_{a}(t)=|1-a|+\frac{t a^{2}}{2} .
$$

From (8), (11) and (14), we get

$$
\begin{aligned}
\left\|\Gamma_{0} F\left(z_{0}\right)\right\| & \leq\left\|\Gamma_{0}\right\|\left\|F\left(z_{0}\right)\right\| \\
& \leq \beta_{0}\left(M|a| \eta_{0} q_{a}\left(a_{0}\right) \eta_{0}+\frac{1}{2} M q_{a}\left(a_{0}\right)^{2} \eta_{0}^{2}\right)=\eta_{0} p_{a}\left(a_{0}\right),
\end{aligned}
$$

where we use the notation

$$
p_{a}(t)=t|a| q_{a}(t)+\frac{t}{2} q_{a}(t)^{2}
$$

Substituting (15) and (13) in (10), one has

$$
\left\|x_{1}-x_{0}\right\| \leq \eta_{0} p_{a}\left(a_{0}\right)+\eta_{0}\left(1+\frac{a_{0}}{2} a^{2}\right)=\eta_{0} g_{a}\left(a_{0}\right) .
$$

where, by definition,

$$
g_{a}(t)=p_{a}(t)+1+\frac{t}{2} a^{2} .
$$

Turning to equation (9), in order to apply Banach's lemma we need

$$
\left\|I-\Gamma_{0} F^{\prime}\left(x_{1}\right)\right\| \leq M \beta_{0}\left\|x_{1}-x_{0}\right\| \leq a_{0} g_{a}\left(a_{0}\right)<1 .
$$


As we will see in Lemma $3, g_{a}(t)$ is increasing, so the polynomial $p(t)=t g_{a}(t)-1$ is unbounded and verifies $p(0)<0$, so that it has a positive root. Let $t_{0}$ be the smallest positive root of $p(t)$. If $\left.a_{0} \in\right] 0, t_{0}\left[\right.$, we have $a_{0} g_{a}\left(a_{0}\right)<1$ and so, we can apply Banach's Lemma 2:

$$
\left\|\left(\Gamma_{0} F^{\prime}\left(x_{1}\right)\right)^{-1}\right\| \leq \frac{1}{1-a_{0} g_{a}\left(a_{0}\right)} .
$$

Then, there exists $\Gamma_{1}=F^{\prime}\left(x_{1}\right)^{-1}$ and

$$
\begin{aligned}
\left\|\Gamma_{1}\right\| & \leq\left\|\Gamma_{1} F^{\prime}\left(x_{0}\right)\right\|\left\|\Gamma_{0}\right\|=\left\|\left(\Gamma_{0} F^{\prime}\left(x_{1}\right)\right)^{-1}\right\|\left\|\Gamma_{0}\right\| \\
& \leq \frac{\left\|\Gamma_{0}\right\|}{1-a_{0} g_{a}\left(a_{0}\right)}=\left\|\Gamma_{0}\right\| f_{a}\left(a_{0}\right),
\end{aligned}
$$

where, by definition,

$$
f_{a}(t)=\frac{1}{1-t g_{a}(t)}
$$

Details on the choice of $a_{0}$ are presented below

Resuming the process, the Taylor expansion of $F\left(x_{1}\right)$ around $z_{0}$ can be written as

$$
\begin{aligned}
F\left(x_{1}\right) & =F\left(z_{0}\right)+F^{\prime}\left(z_{0}\right)\left(x_{1}-z_{0}\right)+\frac{1}{2} F^{\prime \prime}\left(z_{0}\right)\left(x_{1}-z_{0}\right)^{2} \\
& +\int_{0}^{1}\left(F^{\prime \prime}\left(z_{0}+t\left(x_{1}-z_{0}\right)\right)-F^{\prime \prime}\left(z_{0}\right)\right)\left(x_{1}-z_{0}\right)^{2}(1-t) d t .
\end{aligned}
$$

Using (2) and (3), we have

$$
F\left(z_{0}\right)=-F^{\prime}\left(x_{0}\right)\left(x_{1}-z_{0}\right)
$$

and then,

$$
\begin{aligned}
F\left(x_{1}\right)= & \left.\left(F^{\prime}\left(z_{0}\right)-F^{\prime}\left(x_{0}\right)\right)\left(x_{1}-z_{0}\right)+\frac{1}{2} F^{\prime \prime}\left(z_{0}\right)\left(x_{1}-z_{0}\right)\right)^{2} \\
& +\int_{0}^{1}\left(F^{\prime \prime}\left(z_{0}+t\left(x_{1}-z_{0}\right)\right)-F^{\prime \prime}\left(z_{0}\right)\right)\left(x_{1}-z_{0}\right)^{2}(1-t) d t \\
= & \left.\int_{x_{0}}^{z_{0}} F^{\prime \prime}(\tau) d \tau\left(x_{1}-z_{0}\right)+\frac{1}{2} F^{\prime \prime}\left(z_{0}\right)\left(x_{1}-z_{0}\right)\right)^{2}+ \\
& +\int_{0}^{1}\left(F^{\prime \prime}\left(z_{0}+t\left(x_{1}-z_{0}\right)\right)-F^{\prime \prime}\left(z_{0}\right)\right)\left(x_{1}-z_{0}\right)^{2}(1-t) d t
\end{aligned}
$$

Taking norms and using (6) and (7), we can write

$$
\left\|F\left(x_{1}\right)\right\| \leq M\left\|x_{0}-z_{0}\right\|\left\|x_{1}-z_{0}\right\|+\frac{M}{2}\left\|x_{1}-z_{0}\right\|^{2}+\frac{K}{6}\left\|x_{1}-z_{0}\right\|^{3} .
$$

From (2) and (13) we have

$$
\left\|x_{0}-z_{0}\right\|=\left\|\Gamma_{0}\left(F\left(y_{0}\right)+a F\left(x_{0}\right)\right)\right\| \leq \eta_{0}\left(1+\frac{a_{0}}{2} a^{2}\right),
$$

and from (3), (4) and (15),

$$
\left\|x_{1}-z_{0}\right\|=\left\|\Gamma_{0} F\left(z_{0}\right)\right\| \leq p_{a}\left(a_{0}\right) \eta_{0} .
$$


Substituting (21) and (22) in (20) and using (18), we get

$$
\begin{aligned}
& \left\|\Gamma_{1} F\left(x_{1}\right)\right\| \leq\left\|\Gamma_{1}\right\|\left\|F\left(x_{1}\right)\right\| \leq\left\|\Gamma_{0}\right\| f_{a}\left(a_{0}\right)\left\|F\left(x_{1}\right)\right\| \\
& \leq \beta_{0} f_{a}\left(a_{0}\right)\left(M \eta_{0}\left(1+\frac{a_{0}}{2} a^{2}\right) p_{a}\left(a_{0}\right) \eta_{0}+\frac{M}{2} p_{a}\left(a_{0}\right)^{2} \eta_{0}^{2}+\frac{K}{6} p_{a}\left(a_{0}\right)^{3} \eta_{0}^{3}\right) \\
& =f_{a}\left(a_{0}\right)\left(\left(a_{0}+\frac{a_{0}^{2}}{2} a^{2}\right) p_{a}\left(a_{0}\right)+\frac{a_{0}}{2} p_{a}\left(a_{0}\right)^{2}+\frac{b_{0}}{6} p_{a}\left(a_{0}\right)^{3}\right) \eta_{0} \\
& =f_{a}\left(a_{0}\right) \varphi_{a}\left(a_{0}, b_{0}\right) \eta_{0},
\end{aligned}
$$

where, by definition,

$$
\varphi_{a}(t, u)=\left(t+\frac{t^{2}}{2} a^{2}\right) p_{a}(t)+\frac{t}{2} p_{a}(t)^{2}+\frac{u}{6} p_{a}(t)^{3} .
$$

\section{Recurrence relations}

In this section we define the recurrence relations and give some technical lemmas that allow to establish the convergence properties of the iterative method.

Let us consider the following sequences:

$$
\begin{aligned}
& a_{n+1}=a_{n} f_{a}\left(a_{n}\right)^{2} \varphi_{a}\left(a_{n}, b_{n}\right) \\
& b_{n+1}=b_{n} f_{a}\left(a_{n}\right)^{3} \varphi_{a}\left(a_{n}, b_{n}\right)^{2} \\
& \eta_{n+1}=\eta_{n} f_{a}\left(a_{n}\right) \varphi_{a}\left(a_{n}, b_{n}\right),
\end{aligned}
$$

for $n=0,1, \ldots$

Lemma 3 Let $g_{a}, f_{a}$ and $\varphi_{a}$ be the functions defined by (17), (19) and (23), respectively. Suppose $\left.a_{0} \in\right] 0, t_{0}\left[\right.$, where $t_{0}$ is the smallest positive root of the polynomial $t g_{a}(t)-1$ and that $f_{a}\left(a_{0}\right)^{2} \varphi_{a}\left(a_{0}, b_{0}\right)<1$. Then we have,

(i) $g_{a}$ and $f_{a}$ are increasing, and $\left.g_{a}(t)>1, f_{a}(t)>1 \forall t \in\right] 0, t_{0}[$.

(ii) $\varphi_{a}(t, u)$ is increasing as a function of $t$, so is it as a function of $\left.u, \forall t \in\right] 0, t_{0}[$, and $u>0$.

(iii) The sequences $a_{n}, b_{n}$ and $\eta_{n}$ are decreasing and $g_{a}\left(a_{n}\right) a_{n}<1$ as well as $f_{a}\left(a_{n}\right)^{2} \varphi_{a}\left(a_{n}, b_{n}\right)<$ $1, \forall n \geq 0$.

\section{Proof:}

$(i, i i)$ are trivially deduced from the definition of the functions.

(iii) By hypothesis

$$
a_{1}=a_{0} f_{a}\left(a_{0}\right)^{2} \varphi_{a}\left(a_{0}, b_{0}\right)<a_{0}
$$

and

$$
b_{1}=b_{0} f_{a}\left(a_{0}\right)^{3} \varphi_{a}\left(a_{0}, b_{0}\right)^{2}<b_{0} f_{a}\left(a_{0}\right) \varphi_{a}\left(a_{0}, b_{0}\right)<b_{0},
$$

since $f_{a}\left(a_{0}\right)^{2} \varphi_{a}\left(a_{0}, b_{0}\right)<1$ and $f_{a}\left(a_{0}\right)>1$ imply that $f_{a}\left(a_{0}\right) \varphi_{a}\left(a_{0}, b_{0}\right)<1$.

For the sequence $\eta_{n}$ we have

$$
\eta_{1}=\eta_{0} f_{a}\left(a_{0}\right) \varphi_{a}\left(a_{0}, b_{0}\right) \leq \eta_{0}
$$

and by an induction procedure and using that $f_{a}$ and $\varphi_{a}$ are increasing the Lemma holds. 
Lemma 4 Let $p_{a}$ and $\varphi_{a}$ be the functions defined by (16) and (23), respectively. Suppose $\theta \in] 0,1\left[\right.$, then $p_{a}(\theta t)<\theta p_{a}(t)$, and $\varphi_{a}\left(\theta t, \theta^{2} u\right)<\theta^{2} \varphi_{a}(t, u) \forall a \in \mathbb{R}$.

For $a=1$ we have $p_{1}(\theta t)<\theta^{2} p_{1}(t)$, and $\varphi_{1}\left(\theta t, \theta^{2} u\right)<\theta^{3} \varphi_{1}(t, u)$.

Proof: The result is easy to obtain by taking into account the definition of these functions. For $a=1, p_{1}(t)=\frac{t^{2}}{2}+\frac{t^{3}}{8}$.

Lemma 5 Under the hypothesis of Lemma 3, defining $\gamma=f_{a}\left(a_{0}\right)^{2} \varphi_{a}\left(a_{0}, b_{0}\right)$ and $\Delta=\frac{1}{f_{a}\left(a_{0}\right)}$, we have:

(i) $\gamma \in] 0,1[$

For $a \neq 1$ we have:

(ii) $a_{n} \leq \gamma^{3^{n-1}} a_{n-1} \leq \gamma^{\frac{3^{n}-1}{2}} a_{0}$

$b_{n} \leq\left(\gamma^{3^{n-1}}\right)^{2} b_{n-1} \leq \gamma^{\frac{\left(3^{n}-1\right) 2}{2}} b_{0}$

(iii) $f_{a}\left(a_{n}\right) \varphi_{a}\left(a_{n}, b_{n}\right) \leq \gamma^{3^{n}} \Delta, \quad \forall n \in N$

(iv) $\eta_{n} \leq \gamma^{\frac{3^{n}-1}{2}} \Delta^{n} \eta_{0}$ and $\sum_{k=n}^{n+m-1} \eta_{k} \leq \Delta^{n} \gamma^{\frac{3^{n}-1}{2}} \frac{1-\left(\Delta \gamma^{3^{n}}\right)^{m}}{1-\Delta \gamma^{3^{n}}} \eta_{0}$.

For $a=1$ the results are:

(ii') $a_{n} \leq \gamma^{4^{n-1}} a_{n-1} \leq \gamma^{\frac{4^{n}-1}{3}} a_{0}$

$b_{n} \leq\left(\gamma^{4^{n-1}}\right)^{2} b_{n-1} \leq \gamma^{\frac{\left(4^{n}-1\right) 2}{3}} b_{0}$

(iii') $f_{1}\left(a_{n}\right) \varphi_{1}\left(a_{n}, b_{n}\right) \leq \gamma^{4^{n}} \Delta, \quad \forall n \in N$

(iv') $\eta_{n} \leq \gamma^{\frac{4^{n}-1}{3}} \Delta^{n} \eta_{0}$ and $\sum_{k=n}^{n+m-1} \eta_{k} \leq \Delta^{n} \gamma^{\frac{4^{n}-1}{3}} \frac{1-\left(\Delta \gamma^{4^{n}}\right)^{m}}{1-\Delta \gamma^{4^{n}}} \eta_{0}$.

\section{Proof:}

(i) By definition, $\gamma \in] 0,1[$

(ii) $a_{1} \leq \gamma^{4^{0}} a_{0}$

$b_{1}=b_{0} f_{a}\left(a_{0}\right)^{3} \varphi_{a}\left(a_{0}, b_{0}\right)^{2}=b_{0} \gamma f_{a}\left(a_{0}\right) \varphi_{a}\left(a_{0}, b_{0}\right) \leq b_{0} \gamma^{2}$

Let us suppose that the relations hold for $k<n$, then by using Lemma 3 and taking into account that $f_{a}$ is increasing, we have the result for $n$.

If $a \neq 1$, we have

$$
\begin{aligned}
a_{n} & =a_{n-1} f_{a}\left(a_{n-1}\right)^{2} \varphi_{a}\left(a_{n-1}, b_{n-1}\right) \\
& \leq \gamma^{3^{n-2}} a_{n-2} f_{a}\left(\gamma^{3^{n-2}} a_{n-2}\right)^{2} \varphi_{a}\left(\gamma^{3^{n-2}} a_{n-2},\left(\gamma^{3^{n-2}}\right)^{2} b_{n-2}\right) \\
& \leq \gamma^{3^{n-2}} a_{n-2} f_{a}\left(a_{n-2}\right)^{2}\left(\gamma^{3^{n-2}}\right)^{2} \varphi_{a}\left(a_{n-2}, b_{n-2}\right)=\gamma^{3^{n-1}} a_{n-1} .
\end{aligned}
$$

So, it can be established that: $a_{n} \leq \gamma^{3^{n-1}} a_{n-1} \leq \ldots \leq \gamma^{3^{n-1}} \gamma^{3^{n-2}} \ldots \gamma a_{0}=$ $\gamma^{\frac{3^{n}-1}{2}} a_{0}$.

But, for $a=1$, the result is

$$
\begin{aligned}
a_{n} & =a_{n-1} f_{1}\left(a_{n-1}\right)^{2} \varphi_{1}\left(a_{n-1}, b_{n-1}\right) \\
& \leq \gamma^{4^{n-2}} a_{n-2} f_{1}\left(\gamma^{4^{n-2}} a_{n-2}\right)^{2} \varphi_{a}\left(\gamma^{4^{n-2}} a_{n-2},\left(\gamma^{4^{n-2}}\right)^{2} b_{n-2}\right) \\
& \leq \gamma^{4^{n-2}} a_{n-2} f_{1}\left(a_{n-2}\right)^{2}\left(\gamma^{4^{n-2}}\right)^{3} \varphi_{1}\left(a_{n-2}, b_{n-2}\right)=\gamma^{4^{n-1}} a_{n-1} .
\end{aligned}
$$


and then, $a_{n} \leq \gamma^{4^{n-1}} a_{n-1} \leq \ldots \leq \gamma^{4^{n-1}} \gamma^{4^{n-2}} \ldots \gamma a_{0}=\gamma^{\frac{4^{n}-1}{3}} a_{0}$.

A similar reasoning gives the result for $b_{n}$.

We will prove the next items for $a=1$. For $a \neq 1$ the analysis is analogous.

(iii)

$$
\begin{aligned}
f_{1}\left(a_{n}\right) \varphi_{1}\left(a_{n}, b_{n}\right) & \leq f_{1}\left(\gamma^{\frac{4^{n}-1}{3}} a_{0}\right) \varphi_{1}\left(\gamma^{\frac{4^{n}-1}{3}} a_{0}, \gamma^{\frac{\left(4^{n}-1\right) 2}{3}} b_{0}\right) \\
& \leq f_{1}\left(a_{0}\right) \gamma^{4^{n}-1} \varphi_{1}\left(a_{0}, b_{0}\right)=\gamma^{4^{n}} / f_{1}\left(a_{0}\right)
\end{aligned}
$$

(iv) As a direct consequence of iii) we have

$$
\prod_{k=0}^{n-1} f_{1}\left(a_{k}\right) \varphi_{1}\left(a_{k}, b_{k}\right) \leq \prod_{k=0}^{n-1} \frac{\gamma^{4^{k}}}{f_{1}\left(a_{k}\right)} \leq \gamma^{\frac{4^{n}-1}{3}} \Delta^{n}, \forall n \in N
$$

and by definition of $\eta_{n}$ we get:

$$
\eta_{n}=f_{1}\left(a_{n-1}\right) \varphi_{1}\left(a_{n-1}, b_{n-1}\right) \eta_{n-1} \leq \prod_{k=0}^{n-1} f_{1}\left(a_{k}\right) \varphi_{1}\left(a_{k}, b_{k}\right) \eta_{0} \leq \gamma^{\frac{4^{n}-1}{3}} \Delta^{n} \eta_{0},
$$

and then

$$
\begin{gathered}
\sum_{k=n}^{n+m-1} \eta_{k} \leq \sum_{k=n}^{n+m-1} \Delta^{k} \gamma^{\frac{4^{k}-1}{3}} \eta_{0} \leq \gamma^{\frac{4^{n}-1}{3}} \sum_{k=0}^{m-1} \Delta^{n+k}\left(\gamma^{4^{n}}\right)^{\frac{4^{k}-1}{3}} \eta_{0} \\
\leq \Delta^{n} \gamma^{\frac{4^{n}-1}{3}} \sum_{k=0}^{m-1} \Delta^{k}\left(\gamma^{4^{n}}\right)^{k} \eta_{0} \leq \Delta^{n} \gamma^{\frac{4^{n}-1}{3}} \frac{1-\left(\Delta \gamma^{4^{n}}\right)^{m}}{1-\Delta \gamma^{4^{n}}} \eta_{0} .
\end{gathered}
$$

As a consequence of the last item of previous lemma, considering that $\Delta$ and $\gamma$ are less than 1 , we get a convergent series verifying:

$$
\sum_{k=0}^{\infty} \eta_{k} \leq \frac{1}{1-\Delta \gamma} \eta_{0}
$$

Let $R=g_{a}\left(a_{0}\right) \frac{1}{1-\Delta \gamma}$. In what follows we will consider the ball $B\left(x_{0}, R \eta_{0}\right)$ in order to establish the semilocal convergence of the family of iterative methods (3).

Lemma 6 Under the hypothesis of Lemma 3 and the conditions (4-7), the following statements are true for all $n \geq 0$

(I) There exists $\Gamma_{n}$ and $\left\|\Gamma_{n}\right\| \leq f_{a}\left(a_{n-1}\right)\left\|\Gamma_{n-1}\right\|$

(II) $\left\|\Gamma_{n} F\left(x_{n}\right)\right\| \leq \eta_{n}$

(III) $M\left\|\Gamma_{n}\right\|\left\|\Gamma_{n} F\left(x_{n}\right)\right\| \leq a_{n}$

(IV) $N\left\|\Gamma_{n}\right\|\left\|\Gamma_{n} F\left(x_{n}\right)\right\|^{2} \leq b_{n}$

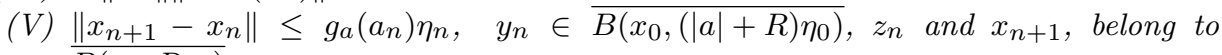
$B\left(x_{0}, R \eta_{0}\right)$

(VI) $R<1 / a_{0}, \forall a \in \mathrm{R}$. 
Proof: Items (I)-(IV) can be easily obtained by the previously described development and an inductive procedure. We now prove item $(\mathrm{V})$ in the following way:

$$
\left\|x_{n+1}-x_{0}\right\| \leq \sum_{i=0}^{n}\left\|x_{i+1}-x_{i}\right\| \leq \sum_{i=0}^{n} g_{a}\left(a_{i}\right) \eta_{i} \leq g_{a}\left(a_{0}\right) \sum_{i=0}^{n} \eta_{i} \leq R \eta_{0}
$$

In a similar way, if $|a| \leq 1$, by construction of $g_{a}$ we have $g_{a}\left(a_{0}\right)>1 \geq|a|$, and so

$$
\left\|y_{n}-x_{0}\right\| \leq\left\|y_{n}-x_{n}\right\|+\left\|x_{n}-x_{0}\right\| \leq|a| \eta_{n}+\sum_{i=0}^{n-1} g_{a}\left(a_{i}\right) \eta_{i} \leq g_{a}\left(a_{0}\right) \sum_{i=0}^{n} \eta_{i} \leq R \eta_{0}
$$

If $|a|>1$,

$$
\begin{aligned}
\left\|y_{n}-x_{0}\right\| & \leq|a| \eta_{n}+\sum_{i=0}^{n-1} g_{a}\left(a_{i}\right) \eta_{i} \leq|a| \eta_{0}+g_{a}\left(a_{0}\right) \sum_{i=0}^{n-1} \eta_{i} \\
& \leq\left(|a|+g_{a}\left(a_{0}\right) \frac{1}{1-\Delta \gamma}\right) \eta_{0} \leq(|a|+R) \eta_{0}
\end{aligned}
$$

so $y_{n} \in B\left(x_{0},(|a|+R) \eta_{0}\right)$.

For the sequence $z_{n}$, using the reasoning given in (13), we have:

$$
\begin{aligned}
\left\|z_{n}-x_{0}\right\| & \leq\left\|z_{n}-x_{n}\right\|+\left\|x_{n}-x_{0}\right\| \\
& \leq\left(1+\frac{a^{2}}{2} a_{n}\right) \eta_{n}+\sum_{i=0}^{n-1} g_{a}\left(a_{i}\right) \eta_{i} \leq g_{a}\left(a_{0}\right) \sum_{i=0}^{n} \eta_{i} \leq R \eta_{0} .
\end{aligned}
$$

In the last inequality we have used that $\left(1+\frac{a^{2}}{2} a_{n}\right) \leq\left(1+\frac{a^{2}}{2} a_{0}\right) \leq g_{a}\left(a_{0}\right)$, what is obvious by the definition of $g_{a}$.

Finally, from the definition of $R, \Delta$ and $\gamma$ can be obtained that:

$$
R=g_{a}\left(a_{0}\right) \frac{1}{1-\Delta \gamma}=\frac{g_{a}\left(a_{0}\right)}{1-f_{a}\left(a_{0}\right) \varphi_{a}\left(a_{0}, b_{0}\right)}<\frac{g_{a}\left(a_{0}\right)}{1-f_{a}\left(a_{0}\right)}=1 / a_{0}
$$

where we have used that $\varphi_{a}\left(a_{0}, b_{0}\right)<1$ from the hypothesis of Lemma 2 and the definition of $f_{a}$ and $g_{a}$.

\section{Semilocal convergence}

At this point, we are going to establish the domain of the existence and uniqueness of the solution, by using the previously obtained results.

Theorem 1 Let $X$ and $Y$ be Banach spaces and $F: \Omega \subseteq X \rightarrow Y$ a nonlinear operator continuously third-order Fréchet differentiable in an open convex subset $\Omega_{0} \subseteq \Omega$. Assume that $x_{0} \in \Omega_{0}$ and that conditions (4-7) hold.

Considering $a_{0}=M \beta \eta$ and $b_{0}=N \beta \eta^{2}$ with $\left.a_{0} \in\right] 0, t_{0}\left[\right.$, where $t_{0}$ is the smallest positive root of the scalar function $\operatorname{tg}_{a}(t)-1$ and the functions $g_{a}, f_{a}$ and $\varphi_{a}$ defined by (17), (19) and (23), respectively. 
If $\overline{B\left(x_{0},(|a|+R) \eta_{0}\right)} \subseteq \Omega$ where $R=g_{a}\left(a_{0}\right) \frac{1}{1-\Delta \gamma}$ with $\Delta=\frac{1}{f_{a}\left(a_{0}\right)}$, and $\gamma=$ $f_{a}\left(a_{0}\right)^{2} \varphi_{a}\left(a_{0}, b_{0}\right)<1$ then, the sequence $\left\{x_{n}\right\}$ defined by (3) converges to a solution $x^{*}$ of $F(x)=0$. The $R$-order of convergence is at least three for any $a \in R$ and for $a=1$ is at least four. The iterates $y_{n} \in \overline{B\left(x_{0},(|a|+R) \eta_{0}\right)}, z_{n}, x_{n+1}$, and $x^{*}$ belong to $\overline{B\left(x_{0}, R \eta_{0}\right)}$ and $x^{*}$ is the unique solution of $F(x)$ in $B\left(x_{0}, 2 /(M \beta)-R \eta_{0}\right) \cap \Omega$.

Moreover, an a priori error estimation can be given, for $a \neq 1$ :

$$
\left\|x_{n}-x^{*}\right\| \leq g_{a}\left(a_{0}\right) \Delta^{n} \gamma^{\frac{3^{n}-1}{2}} \frac{1}{1-\Delta \gamma^{3^{n}}} \eta_{0}
$$

and, for $a=1$ :

$$
\left\|x_{n}-x^{*}\right\| \leq g_{1}\left(a_{0}\right) \Delta^{n} \gamma^{\frac{4^{n}-1}{3}} \frac{1}{1-\Delta \gamma^{4^{n}}} \eta_{0} .
$$

Proof: The iterative process is well defined as we have proved in the previous Lemmas.

Now we prove that $x_{n}$ is a Cauchy sequence by using that $g_{a}$ is increasing and $a_{k} \leq a_{n} \forall k \leq n$, so that $g_{a}\left(a_{k}\right) \leq g_{a}\left(a_{n}\right)$ and, by Lemma 3 (iv),

$$
\begin{aligned}
\left\|x_{n+m}-x_{n}\right\| & \leq \sum_{i=n}^{n+m-1}\left\|x_{i+1}-x_{i}\right\| \leq \sum_{i=n}^{n+m-1} g_{a}\left(a_{i}\right) \eta_{i} \\
& \leq g_{a}\left(a_{0}\right) \sum_{i=n}^{n+m-1} \eta_{i} \leq g_{a}\left(a_{0}\right) \Delta^{n} \gamma^{\frac{3^{n}-1}{2}} \frac{1-\left(\Delta \gamma^{3^{n}}\right)^{m}}{1-\Delta \gamma^{3^{n}}} \eta_{0}
\end{aligned}
$$

so that, $\left\{x_{n}\right\}$ is a Cauchy sequence, and then it has a limit $x^{*}$. By taking $m \rightarrow \infty$ we obtain an a priori error estimation:

$$
\left\|x_{n}-x^{*}\right\| \leq g_{a}\left(a_{0}\right) \Delta^{n} \gamma^{\frac{3^{n}-1}{2}} \frac{1}{1-\Delta \gamma^{3^{n}}} \eta_{0}
$$

and taking $n=0$ in (28) and $m \rightarrow \infty$, we have

$$
\left\|x^{*}-x_{0}\right\| \leq R \eta_{0}
$$

Then $x^{*} \in \overline{B\left(x_{0}, R \eta_{0}\right)}$. Moreover $x^{*}$ is a solution of $F(x)=0$ since

$$
\begin{aligned}
\left\|F^{\prime}\left(x_{n}\right)\right\| & \leq\left\|F^{\prime}\left(x_{0}\right)\right\|+\left\|F^{\prime}\left(x_{n}\right)-F^{\prime}\left(x_{0}\right)\right\| \\
& \leq\left\|F^{\prime}\left(x_{0}\right)\right\|+M\left\|x_{n}-x_{0}\right\| \leq\left\|F^{\prime}\left(x_{0}\right)\right\|+M R \eta_{0} .
\end{aligned}
$$

$F^{\prime}\left(x_{n}\right)$ is bounded and, using Lemma 5 (iv), one has $\left\|\Gamma_{n} F\left(x_{n}\right)\right\| \rightarrow 0$, so we can establish that

$$
\left\|F\left(x_{n}\right)\right\| \leq\left\|F^{\prime}\left(x_{n}\right)\right\|\left\|\Gamma_{n} F\left(x_{n}\right)\right\| \rightarrow 0
$$

and, by the continuity of $F$, we get $F\left(x^{*}\right)=0$.

Now, to prove the uniqueness. Let us suppose that $y^{*} \in B\left(x_{0}, 2 / M \beta-R \eta_{0}\right) \cap \Omega_{0}$ is another solution of $F(x)=0$. Then,

$$
0=F\left(y^{*}\right)-F\left(x^{*}\right)=\int_{0}^{1} F^{\prime}\left(x^{*}+t\left(y^{*}-x^{*}\right)\right) d t\left(y^{*}-x^{*}\right) .
$$


Using the following estimation

$$
\begin{aligned}
& \left\|\Gamma_{0}\right\| \int_{0}^{1}\left\|F^{\prime}\left(x^{*}+t\left(y^{*}-x^{*}\right)\right)-F^{\prime}\left(x_{0}\right)\right\| d t \\
& \leq M \beta \int_{0}^{1}\left\|x^{*}+t\left(y^{*}-x^{*}\right)-x_{0}\right\| d t \\
& \leq M \beta \int_{0}^{1}\left((1-t)\left\|x^{*}-x_{0}\right\|+t\left\|y^{*}-x_{0}\right\|\right) d t \\
& <(M \beta / 2)\left(R \eta_{0}+2 / M \beta-R \eta_{0}\right)=1,
\end{aligned}
$$

we can apply Banach's Lemma, (2) and then, the operator $\int_{0}^{1} F^{\prime}\left(x^{*}+t\left(y^{*}-x^{*}\right)\right) d t$ has an inverse and, consequently, $y^{*}=x^{*}$.

\section{Note}

Here we study the conditions of applicability of the methods, needed for applying Banach's lemma in (9): $a_{0} g_{a}\left(a_{0}\right)<1$ and the ones imposed in lemma 2 , that is, $\Delta, \gamma<1$.

Function $g_{a}(t)$ defined in (17) can be expressed as

$$
g_{a}(t)=1+\frac{1}{2}(|a|+|1-a|)^{2} t+\frac{1}{2}(|a|+|1-a|) a^{2} t^{2}+\frac{1}{8} a^{4} t^{3} .
$$

Notating $d=|a|+|1-a|$, it is obvious that $1 \leq d$ and $|a| \leq d$. Taking $0<t<\frac{1}{2 d}$ one has

$$
0<t g_{a}(t)=t+\frac{1}{2} d^{2} t^{2}+\frac{1}{2} d a^{2} t^{3}+\frac{1}{8} a^{4} t^{4}<\frac{1}{2}+\frac{1}{2} \frac{1}{4}+\frac{1}{2} \frac{1}{2} \frac{1}{4}+\frac{1}{8} \frac{1}{16}<1 .
$$

Particularly, for $0 \leq a \leq 1, \quad d=1$ and then, $t g_{a}(t)<1$ for $t \in\left[0, \frac{1}{2}\right]$.

In general, if $a_{0} \leq \frac{1}{2 d}$, Banach's lemma can be applied and $\Delta<1$.

On the other hand, $\gamma<1$ if $b_{0}$ satisfies the following condition derived from (23):

$$
b_{0}<\frac{6}{p_{a}\left(a_{0}\right)^{3}}\left(\frac{1}{f_{a}\left(a_{0}\right)^{2}}-\left(a_{0}+\frac{a_{0}^{2}}{2} a^{2}\right) p_{a}\left(a_{0}\right)-\frac{a_{0}}{2} p_{a}\left(a_{0}\right)^{2}\right) .
$$

Using this relation, we can determine bounds for $a_{0}$ and $b_{0}$ depending on the parameter $a$, so that $\gamma<1$. For example, taking $a_{0}<A_{0}=0.95 \min \left(\frac{1}{2 d}, \frac{1}{2 d^{2}}\right)$ and $b_{0}<B_{0}=$ $\frac{1}{2 d}-A_{0}$, one has $\gamma<1$. Figure 1 shows the values of $\gamma$ obtained from the bounds $A_{0}$ and $B_{0}$ corresponding to values $-3 \leq a \leq 4$.

\section{Application example}

In this section we present some numerical data to illustrate the theoretical results, by obtaining the terms of the recurrence relations of our methods, denoted by $N M$, for different values of the parameter $a$. 


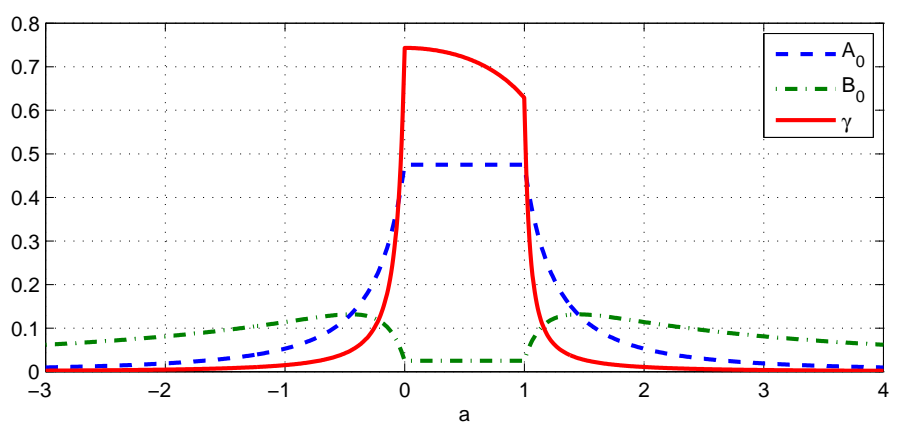

Fig. 1 Bounds for $a_{0}$ and $b_{0}$ that guarantee that $\gamma<1$ for different values of $a$.

We also obtain the value of the convergence and uniqueness radii and compare them with the corresponding to the following recently published methods.

The first one, presented in [11], with R-order at least 4, will be denoted by $\mathrm{M}_{4}$;

$$
\begin{aligned}
K_{F}\left(x_{n}\right) & =\Gamma_{n} F^{\prime \prime}\left(x_{n}-\frac{1}{3} \Gamma_{n} F\left(x_{n}\right)\right) \Gamma_{n} F\left(x_{n}\right) \\
\Delta_{n} & =I-K_{F}\left(x_{n}\right) \\
x_{n+1} & =x_{n}-\left(I+\frac{1}{2} K_{F}\left(x_{n}\right) \Delta_{n}^{-1}\right) \Gamma_{n} F\left(x_{n}\right)
\end{aligned}
$$

The second one, studied in [13], with R-order at least 5 , will be denoted by $\mathrm{M}_{5}$ :

$$
\begin{aligned}
y_{n} & =x_{n}-\Gamma_{n} F\left(x_{n}\right) \\
z_{n} & =x_{n}-\left[1+\frac{1}{2} L_{F}\left(x_{n}\right)\left(I-L_{F}\left(x_{n}\right)^{-1}\right] \Gamma_{n} F\left(x_{n}\right)\right. \\
x_{n+1} & =z_{n}-\left[I+F^{\prime \prime}\left(x_{n}\right) \Gamma_{n}\left(z_{n}-x_{n}\right)\right]^{-1} \Gamma_{n} F\left(z_{n}\right),
\end{aligned}
$$

where $L_{F}\left(x_{n}\right)=\Gamma_{n} F^{\prime \prime}\left(x_{n}\right) \Gamma_{n} F\left(x_{n}\right)$.

The above mentioned methods are compared by applying them to the following examples:

Example 1: (See [13])

Consider the equation $F(x)=0$ given by:

$$
F(x)=\left\{\begin{array}{c}
x^{3}-2 x-5, x \geq 0 \\
-x^{3}-2 x-13, x<0
\end{array}\right.
$$

where $x \in[-1,3]$. It easy to obtain for $x_{0}=2$ the following bounds: $\beta_{0}=0.1, \eta_{0}=0.1$, $M=18$ and $K=6$. Consequently, we obtain the convergence and uniqueness radii given in Table 6 . The behavior of the different methods is similar, but our family does not use the second derivative in its iterative expression.

Example 2: (See [11])

Let $X=C[0,1]$ be the space of continuous functions on $[0,1]$ with the $\infty$-norm,

$$
\|x\|=\max _{s \in[0,1]}|x(s)| .
$$




\begin{tabular}{|c|c|c|c|c|c|}
\hline & $\mathrm{M}_{4}$ & $\mathrm{M}_{5}$ & $\mathrm{NM}_{3} \quad a=0$ & $\mathrm{NM}_{3} \quad a=0.5$ & $\mathrm{NM}_{4} \quad a=1$ \\
\hline Convergence & 0.111153 & 0.138566 & 0.111345 & 0.111300 & 0.111158 \\
\hline Uniqueness radius & 0.999958 & 0.972545 & 0.999766 & 0.999811 & 0.999954 \\
\hline
\end{tabular}

Table 1 Numerical results for Example 1 with starting condition $x_{0}=2$.

Considering the nonlinear integral equation $\mathrm{F}(\mathrm{x})=0$ where

$$
F(x)(s)=x(s)-1+\frac{1}{2} \int_{0}^{1} s \cos (x(t)) d t
$$

where $s \in[0,1]$ and $x \in \Omega=B(0,2) \subset X$.

The derivatives of $F$ are

$$
F^{\prime}(x) y(s)=y(s)-\frac{1}{2} \int_{0}^{1} s \sin (x(t)) y(t) d t
$$

and

$$
F^{\prime \prime}(x) y z(s)=-\frac{1}{2} \int_{0}^{1} s \cos (x(t)) y(t) z(t) d t
$$

for $y, z \in \Omega$.

The second derivative $F^{\prime \prime}$ satisfies

$$
\left\|F^{\prime \prime}(x)\right\| \leq \frac{1}{2}=M, \quad x \in \Omega,
$$

and the Lipschitz condition

$$
\left\|F^{\prime \prime}(x)-F^{\prime \prime}(y)\right\| \leq \frac{1}{2}\|x-y\|, \quad x, y \in \Omega,
$$

so that $K=\frac{1}{2}$.

Starting from an initial estimation $x_{0}(t)=1$ of the solution, we have

$$
\left\|F\left(x_{0}\right)\right\|=\left\|\frac{1}{2} \int_{0}^{1} s \cos \left(x_{0}(t)\right) d t\right\| \leq \frac{1}{2} \cos 1 .
$$

Since

$$
\left\|I-F^{\prime}\left(x_{0}\right)\right\|=\left\|\frac{1}{2} \int_{0}^{1} s \sin \left(x_{0}(t)\right) d t\right\| \leq \frac{1}{2} \sin 1,
$$

it follows by the Banach lemma that $\Gamma_{0}$ exists and

$$
\left\|\Gamma_{0}\right\| \leq \frac{2}{2-\sin 1}=\beta_{0} .
$$

Then,

$$
\left\|\Gamma_{0} F\left(x_{0}\right)\right\| \leq \frac{\cos 1}{2-\sin 1}=\eta_{0} .
$$

The terms of the recurrence relations of the method for different values of the parameter $a$ are shown in Table 2. The convergence speed is almost the same for $a=0,0.5$, but is notably faster in the case $a=1$. Although the values of the radii are quite similar, the best results are obtained by the fourth order method introduced in our work for $a=1$. 


\begin{tabular}{lllll}
\hline$a$ & $n$ & $a_{n}$ & $b_{n}$ & $\eta_{n}$ \\
\hline & 0 & 0.402553 & 0.187738 & 0.466369 \\
& 1 & 0.134994 & 0.0109028 & 0.0807655 \\
& 2 & 0.00173585 & $1.54296 \mathrm{e}-006$ & 0.000888882 \\
0 & 3 & $2.62545 \mathrm{e}-009$ & $3.52357 \mathrm{e}-018$ & $1.34209 \mathrm{e}-009$ \\
& 4 & $9.04855 \mathrm{e}-027$ & $4.18539 \mathrm{e}-053$ & $4.62548 \mathrm{e}-027$ \\
& 5 & $3.70431 \mathrm{e}-079$ & $7.01443 \mathrm{e}-158$ & $1.89359 \mathrm{e}-079$ \\
& 6 & $2.54152 \mathrm{e}-236$ & 0 & $1.29918 \mathrm{e}-236$ \\
\hline & 0 & 0.402553 & 0.187738 & 0.466369 \\
& 1 & 0.122733 & 0.00886641 & 0.0722414 \\
& 2 & 0.000992368 & $5.04012 \mathrm{e}-007$ & 0.000507888 \\
0.5 & 3 & $3.67444 \mathrm{e}-010$ & $6.90312 \mathrm{e}-020$ & $1.87869 \mathrm{e}-010$ \\
& 4 & $1.86039 \mathrm{e}-029$ & $1.76958 \mathrm{e}-058$ & $9.51191 \mathrm{e}-030$ \\
& 5 & $2.41458 \mathrm{e}-087$ & $2.98091 \mathrm{e}-174$ & $1.23454 \mathrm{e}-087$ \\
& 6 & $5.27907 \mathrm{e}-261$ & 0 & $2.69912 \mathrm{e}-261$ \\
\hline \multirow{4}{*}{1} & 0 & 0.402553 & 0.187738 & 0.466369 \\
& 1 & 0.0780124 & 0.00338805 & 0.0434296 \\
& 2 & $2.32772 \mathrm{e}-005$ & $2.77113 \mathrm{e}-010$ & $1.19049 \mathrm{e}-005$ \\
& 3 & $1.46798 \mathrm{e}-019$ & $1.10211 \mathrm{e}-038$ & $7.50768 \mathrm{e}-020$ \\
& 4 & $2.32192 \mathrm{e}-076$ & $2.75728 \mathrm{e}-152$ & $1.1875 \mathrm{e}-076$ \\
& 5 & $1.45332 \mathrm{e}-303$ & 0 & $7.43269 \mathrm{e}-304$ \\
\hline
\end{tabular}

Table 2 Recurrence relations for NM with $a=0,0.5,1$ in Example 2.

\begin{tabular}{|c|c|c|c|c|c|}
\hline & $\mathrm{M}_{4}$ & $\mathrm{M}_{5}$ & $\mathrm{NM}_{3} \quad a=0$ & $\mathrm{NM}_{3} \quad a=0.5$ & $\mathrm{NM}_{4} \quad a=1$ \\
\hline Convergence radius & 0.712479 & 1.305052 & 0.677582 & 0.674387 & 0.663627 \\
Uniqueness radius & 1.604579 & 1.012006 & 1.639477 & 1.642671 & 1.653431 \\
\hline \hline
\end{tabular}

Table 3 Numerical results for Example 2 with starting condition $x_{0}(t)=1$.

\subsection{Computational efficiency}

In order to compare different methods, we use the efficiency and operational indexes, $I$ and $C$ respectively ([1]). That is, $I=p^{1 / d}$, where $p$ is the order of convergence and $d$ is the total number of new functional evaluations (per iteration) required by the method. Also, $C=p^{1 / o p}$, where $o p$ is the number of operations per iteration. We recall that the number of products and quotients that we need for solving $m$ linear systems with the same matrix of coefficient, by using $L U$ factorization, is

$$
\frac{1}{3} n^{3}+m n^{2}-\frac{1}{3} n
$$

where $n$ is the size of each system.

We do not compute the efficiency of the fifth order method described in (30) because the use of second derivatives in the last two steps makes it inefficient. However we compare our methods with Newton's method, N, and a classical method of fourth order for nonlinear systems, Jarratt's method, [22], that we denote by JM and whose iterative expression is:

$$
\begin{aligned}
y_{n} & =x_{n}-\frac{2}{3} \Gamma_{n} F\left(x_{n}\right) \\
x_{n+1} & =x_{n}-\frac{1}{2} \Gamma_{n} F\left(x_{n}\right)\left[\left(3 F^{\prime}\left(y_{n}\right)+F^{\prime}\left(x_{n}\right)\right)^{-1}\left(3 F^{\prime}\left(y_{n}\right)-F^{\prime}\left(x_{n}\right)\right)\right],
\end{aligned}
$$


Notice that both efficiency and operational indexes of Jarrat's method coincide with the ones of Newton's method, since Jarrat's method doubles both the order and the number of functional evaluations and linear systems of Newton's method.

Tables 4 and 5 show the efficiency and operational indices for different sizes of the nonlinear system. Notice that, in terms of $I, \mathrm{NM}_{4}$ is the most efficient, and for $n \geq 2$, the method $\mathrm{NM}_{3}$ is also more efficient than Newton's and Jarrat's method. Similar behavior can be observed in terms of $C$ for bigger values of $n$, so this family of methods is very competitive.

\begin{tabular}{|c|c|c|c|c|}
\hline & $\mathrm{JM}$ & $\mathrm{NM}_{3}$ & $\mathrm{NM}_{4}$ & $\mathrm{M}_{4}$ \\
\hline$I$ & $4^{\frac{1}{2 n+2 n^{2}}}$ & $3^{\frac{1}{3 n+n^{2}}}$ & $4^{\frac{1}{3 n+n^{2}}}$ & $4^{\frac{1}{n+n^{2}+n^{3}}}$ \\
\hline \hline$n=2$ & 1.12246 & 1.11612 & 1.14870 & 1.10409 \\
$n=3$ & 1.05946 & 1.06294 & 1.08006 & 1.03619 \\
$n=4$ & 1.03526 & 1.04002 & 1.05076 & 1.01664 \\
$n=5$ & 1.02337 & 1.02785 & 1.03526 & 1.00898 \\
$n=10$ & 1.00632 & 1.00849 & 1.01072 & 1.00125 \\
$n=20$ & 1.00165 & 1.00239 & 1.00302 & 1.00016 \\
$n=30$ & 1.00075 & 1.00111 & 1.00140 & 1.00005 \\
\hline \hline
\end{tabular}

Table 4 Efficiency indices for different values of $n$.

\begin{tabular}{|c|c|c|c|c|}
\hline & $\mathrm{JM}$ & $\mathrm{NM}_{3}$ & $\mathrm{NM}_{4}$ & $\mathrm{M}_{4}$ \\
\hline \hline$n=2$ & 1.12246 & 1.08163 & 1.10409 & 1.04427 \\
$n=3$ & 1.04162 & 1.03189 & 1.04040 & 1.01439 \\
$n=4$ & 1.01944 & 1.01629 & 1.02060 & 1.00644 \\
$n=5$ & 1.01072 & 1.00960 & 1.01213 & 1.00343 \\
$n=10$ & 1.00161 & 1.00175 & 1.00220 & 1.00047 \\
$n=20$ & 1.00023 & 1.00028 & 1.00036 & 1.00006 \\
$n=30$ & 1.00007 & 1.00009 & 1.00012 & 1.00002 \\
\hline \hline
\end{tabular}

Table 5 Operational indices for different values of $n$.

Finally we discretize the integral equation given in Example 2 in order to have a finite dimensional problem of big size. For this purpose we use the Simpson quadrature formula, with $n$ subintervals so $h=1 / n$, nodes $t_{i}=i h$ with $i=0, \ldots, n$; and weights $p=h / 3(1,4,2, \ldots, 2,4,1) \in \mathbb{R}^{n+1}$. By denoting $x\left(t_{i}\right)=x_{i}$, we have the nonlinear system of equations:

$$
x_{i}-1+\frac{t_{i}}{2} \sum_{j=0}^{n} p_{j} \cos \left(x_{j}\right)=0 ; \quad i=0, \ldots, n .
$$

Table 6 shows the results for different methods, with $n=100$, using variable precision arithmetics that uses floating point representation of 100 decimal digits of mantissa in MATLAB 2010. We calculate the number of iterations and the estimated order of convergence $p,([23])$, for the stopping criterion $\left\|x_{k+1}-x_{k}\right\| \leq 10^{-10}$, the value 
$\left\|F\left(x^{(k)}\right)\right\|$ at this iteration and the average time in seconds, $T$, for 20 runs of the methods. As it can be observed, the new methods reach always similar o better results than classical ones.

\begin{tabular}{|c|c|c|c|c|c|}
\hline & iter & $\left\|x_{n+1}-x_{n}\right\|$ & $\left\|F\left(x_{n+1}\right)\right\|$ & $p$ & $\mathrm{~T}$ \\
\hline \hline$N$ & 6 & $5.4333 \mathrm{e}-51$ & $3.143 \mathrm{e}-103$ & 2.0000 & 672.9568 \\
$J$ & 4 & $1.9847 \mathrm{e}-90$ & $6.3978 \mathrm{e}-108$ & 4.0000 & 876.9897 \\
$H M T(a=1)$ & 4 & $1.0581 \mathrm{e}-90$ & $6.7394 \mathrm{e}-108$ & 4.0000 & 545.4744 \\
$H M T(a=-1)$ & 4 & $6.1919 \mathrm{e}-79$ & $6.1963 \mathrm{e}-108$ & 4.0000 & 551.5068 \\
$H M T(a=0.5)$ & 4 & $1.3892 \mathrm{e}-40$ & $6.9903 \mathrm{e}-108$ & 3.0000 & 549.9432 \\
$H M T(a=0)$ & 4 & $5.8121 \mathrm{e}-39$ & $8.0058 \mathrm{e}-108$ & 3.0000 & 549.6305 \\
\hline
\end{tabular}

Table 6 Starting guess $x_{0}=(1,1, \ldots, 1)$.

\section{Global convergence}

In this section we study the dynamics of the proposed iterative method $\mathrm{NM}_{3}$ with $a=0.5$ when applied to the solution of a system of quadratic equations, representing the intersection of two conics in $\mathbb{R}^{2}$ and compare it with the dynamics of Newton's method. The behavior of the method for other values of parameter $a$ is quite similar.

Let us first recall some dynamical concepts. Consider a Frechet differentiable function $G: \mathbb{R}^{n} \longrightarrow \mathbb{R}^{n}$.

For $x \in \mathbb{R}^{n}$, we define the orbit of $x$ as the set $x, G(x), G^{2}(x), \ldots, G^{p}(x), \ldots$ A point $x_{f}$ is a fixed point of $G$ if $G\left(x_{f}\right)=x_{f}$. A fixed point $x_{f}$ is called attracting if $\left\|J_{G}\left(x_{f}\right)\right\|<1$, repelling if $\left\|J_{G}\left(x_{f}\right)\right\|>1$, and neutral if $\left\|J_{G}\left(x_{f}\right)\right\|=1$. If $J_{G}\left(x_{f}\right)=0$, the point $x_{f}$ is superattracting. Let $x_{a f}$ be an attracting fixed point of the function $\mathrm{G}$. The basin of attraction of $x_{a f}$ is the set of points whose orbits tend to this fixed point

$$
\mathcal{A}\left(x_{a f}\right)=\left\{x \in \mathbb{R}^{n}: G^{p}(x) \longrightarrow x_{a f} \text { for } p \longrightarrow \infty\right\}
$$

The chosen example presents three simple real roots that are superattractive fixd points for the method $\mathrm{NM}_{3}$. We show that the method is generally convergent and depict the attraction basins.

$$
\left.\begin{array}{rl}
x^{2}+2 y & =3 \\
2 x y & =1
\end{array}\right\}
$$

For the comparisons, we have run the methods iterating with tolerance $10^{-12}$ performing a maximum of 50 iterations. The starting points form a uniform grid of $600 \times 600$ in a rectangle of the real plane. The attraction basins have been colored according to the corresponding fixed point.

Figures 2 and 3 show the attraction basins of Newton's method and our method, respectively. The basins of our method are slightly more complex than that of Newton's method, but the convergence regions cover almost all the plane.

Figures 4 and 5 show the difference in convergence speed between Newton's method and $\mathrm{NM}_{3}$, with $a=0.5$. The colored zones correspond to the initial points that attain 


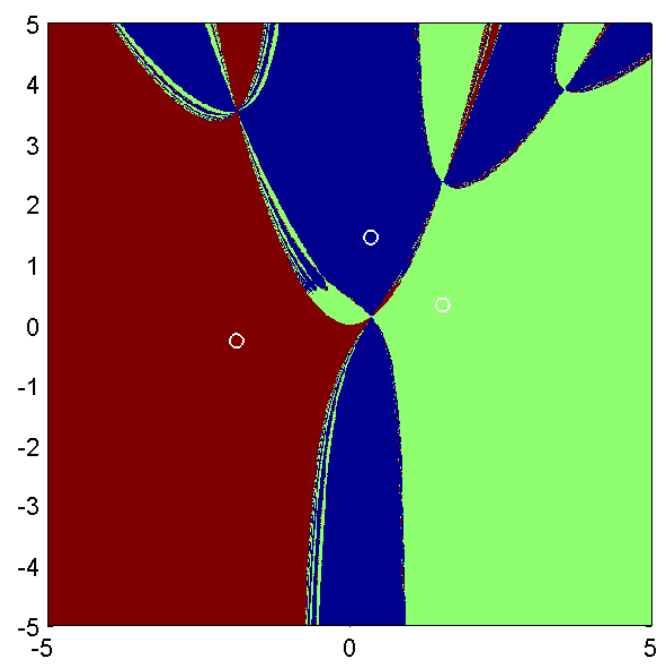

Fig. 2 Attraction basins for Newton's method

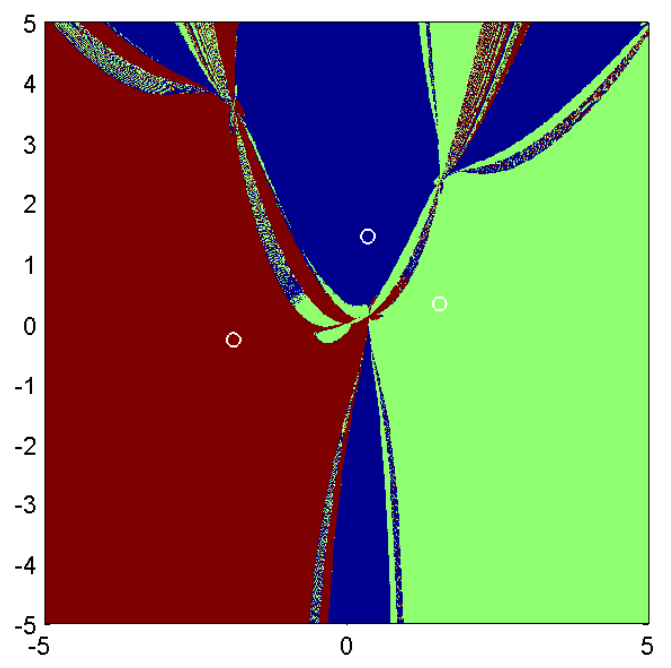

Fig. 3 Attraction basins for method $\mathrm{NM}_{3}, \mathrm{a}=0.5$ 


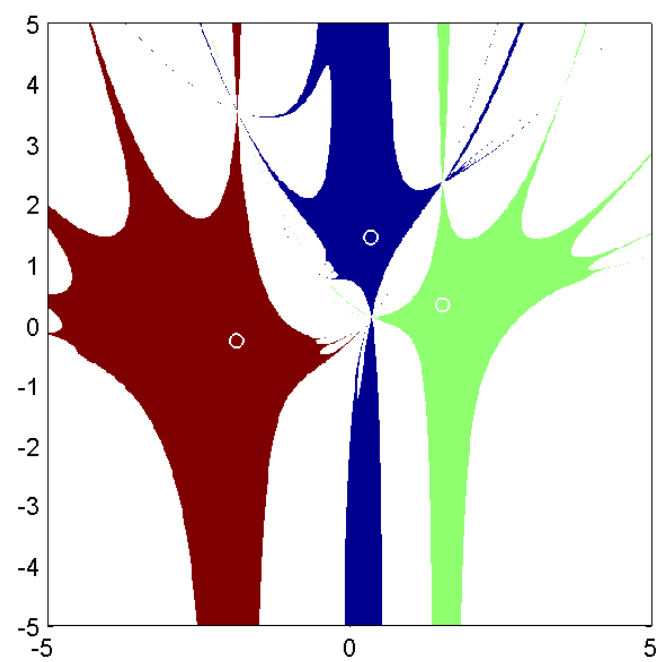

Fig. 4 Points that converge $\left(\right.$ tol $\left.=10^{-12}\right)$ in 5 steps with Newton's method

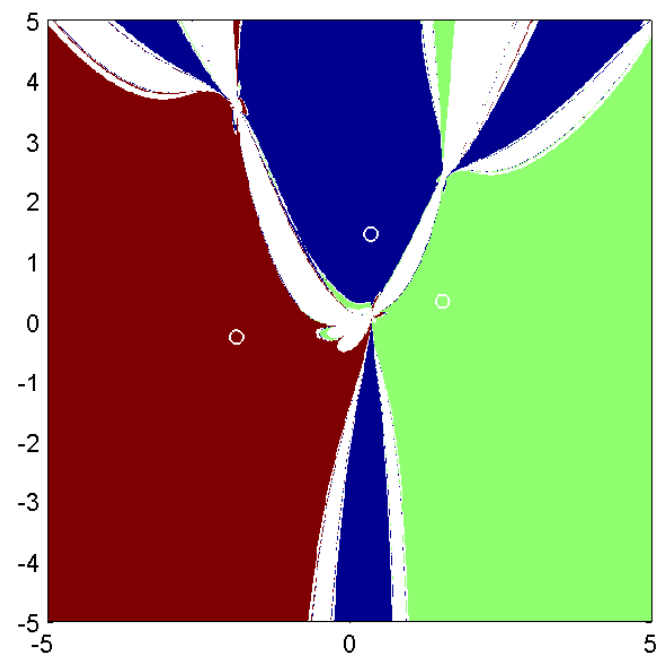

Fig. 5 Points that converge $\left(\right.$ tol $\left.=10^{-12}\right)$ in 5 steps with method $\mathrm{NM}_{3}, \mathrm{a}=0.5$. 
a solution with the required tolerance in 5 iterations. For method $\mathrm{NM}_{3}$, these regions are wider corresponding to the higher convergence order of this method with respect to Newton's method.

\section{Conclusions}

In this paper we establish the semilocal convergence for a family of iterative methods in Banach spaces by constructing the system of recurrence relations and obtaining a priori error estimations. The efficiency indices of these new methods and the numerical results show that these methods are competitive.

The dynamical behavior of the proposed methods has been compared with that of Newton's method. The attraction basins of the new methods are slightly more complex than that of Newton's method, but the convergence is faster as expected due to the difference in convergence orders.

\section{Acknowledgement}

The authors would like to thank the referees for the valuable comments and for the suggestions to improve the readability of the paper.

\section{References}

1. J.F. Traub, Iterative methods for the solution of nonlinear equations, Prentice Hall, New York, 1964.

2. Kantorovich, L. V., On the Newton method for Funtional Equations, Doklady Akademii Nauk SSSR, 59, (1948), 1237-1240.

3. Candela, V., Marquina, A., Recurrence Relations for Rational Cubic Methods, I: The Halley Method, Computing, 44, (1990), 169-184.

4. Candela, V., Marquina, A., Recurrence Relations for Rational Cubic Methods, II: The Chebyshev Method, Computing, 45, (1990), 355-367.

5. Hernández, M.A., Reduced Recurrence Relations for the Chebyshev Method, Journal of Optimization Theory and Applications, 98, (1998), 385-397.

6. Gutiérrez, J.M., Hernández, M.A., Recurrence Relations for super-Halley Method, Journal of Computational and Mathematics Applied, 7, (1998), 1-8.

7. Ezquerro, J.A., Hernández, M.A., Recurrence Relations for Chebyshev-like Methods, Applied Mathematics and Optimization, 41, (2000), 227-236.

8. Ezquerro, J.A., Hernández, M.A., New iterations of $R$-order four with reduced computational cost, BIT Numer. Math., 49, (2009), 325-342.

9. Argyros, I,K., Ezquerro, J.A., Gutiérrez, J.M., Hernández, M.A., Hilout, S., On the semilocal convergence of efficient Chebyshev Secant-type methods, Journal of Computational and Applied Mathematics, 235-10, (2011), 3195-3206.

10. Argyros I. K., Hilout S. Weaker conditions for the convergence of Newtons method Journal of Complexity, 28,3, (2012), 364-387.

11. Wang, X., Gu, C., Kou, J. Semilocal convergence of a multipoint fourth-order super-Halley method in Banach spaces, Numerical Algorithms, 54, (2011), 497516

12. Kou, J., Li, Y., Wang, X., A variant of super Halley method with accelerated fourth-order convergence, Applied Mathematics and Computations, 186, (2007), 535-539.

13. Zheng, L., Gu, C., Recurrence relations for semilocal convergence of a fifth-order method in Banach spaces, Numerical Algorithms, 59, (2012), 623-638.

14. Amat, S., Hernández, M.A., Romero, N., A modified Chebyshevs iterative method with at least sixth order of convergence, Applied Mathematics and Computation, 206, (2008), 164-174. 
15. Wang, X., Kou, J., Gu, C., Semilocal convergence of a sixth-order Jarratt method in Banach spaces, Numerical Algorithms, 57, (2011), 441-456.

16. Hernández, M.A., The Newton Method for Operators with Hlder Continuous First Derivative, Journal of Optimization and Applications, 109, (2001), 631-648.

17. Ye, X., Li, C., Convergence of the family of the deformed Euler-Halley iterations under the Hlder condition of the second derivative, Journal of Computational and Applied Mathematics 194, (2006), 294-308 .

18. Zhao, Y., Wu, Q., Newton-Kantorovich theorem for a family of modified Halleys method under Hlder continuity conditions in Banach spaces, Applied Mathematics and Computations, 202, (2008), 243-251.

19. Argyros, I.K., Improved generalized differentiability conditions for Newton-like methods, Journal of Complexity, 26, (2010), 316-333.

20. Hueso, J.L., Martínez, E., Torregrosa, J.R., Third and fourth order iterative methods free from second derivative for nonlinear systems, Applied Mathematics and Computation, 211, (2009), 190-197.

21. Taylor, A.Y., Lay, D. Introduction to Functional Analysis, 2 Ed. New York. J. Wiley 1980

22. P. Jarrat, Some fourth order multipoint iterative methods for solving equations, Math. Comp., 20, 434-437, (1966).

23. A. Cordero, Juan. R. Torregrosa, Variants of Newtons Method using fifth-order quadrature formulas, Applied Mathematics and Computation, 190, (2007), 686-698. 